\title{
Influencia del trabajo de parto en el equilibrio ácido básico de recién nacido
}

\author{
M.C. Hemán González L.' ; M.C. Carlos Fernández D. ${ }^{2}$ \\ M.C. Braulio Herrera $G{ }^{2}$

\section{Effect of labor on the acid base status of the normal newborn infant}

\begin{abstract}
The vahues of $\mathrm{pH}, \mathrm{PCO}_{2}, \mathrm{PO}_{2}$ and base excess were measured at the time of birth, from the umbilical artery, in a group of 19 normal newborn infants from vaginal deliveries and compared to 19 infants born by etective cesarean sections without labor. The value of $\mathrm{pH}$ was significantly lower $(7.23 \pm 0.06)$ in vaginal deliveries compared to elective cesarean sections $(7.27 \pm 0.03) \mathrm{p}<0.01$. The best correlations ocured between $\mathrm{pH}$ and $\mathrm{PCO}_{2}(\mathrm{I}: 0.72 \mathrm{p}<0.01)$ in vaginal deliveries and $\mathrm{pH}$ and base excess $(0.73 \mathrm{p}<0.01)$ in cesarean sections. Normal values for umbilical arterial $\mathrm{pH}$ should consider the influence of labor in the acid base balance of the newborn infant. We consider that $\mathrm{pH}$ value under 7.11 (mean $-2 \mathrm{SD}$ ) in vaginal deliveries and under 7.21 in elective cesaream sections are indicative of neonatal acidosis.
\end{abstract}

(Key words: umbilical arterial pH, normal vaginal delivery, cesarean section.)

Las mediciones relativas al metabolismo de ácidos y bases en el momento de nacer constituyen uno de los métodos que últimamente se han utilizado en la evaluación del grado de asfixia intrauterina ${ }^{1-4}$.

En general se ha observado correlación entre el $\mathrm{pH}$ medido en la sangre de la arteria umbilical y la condición clínica del recién nacido (RN), evaluada según la puntuación de Apgar ${ }^{5,6}$, observándose valores de $\mathrm{pH}$ más bajos en aquellos recién nacióos que nacen clínicamente deprimidos. Sin embargo, existe un porcentaje importante de recién nacidos ${ }^{2}$ cuyos Apgar no reflejan el estado del equilibrio ácido básico fetal. Esto se debe en parte a la diversidad de criterios para seleccionar a las madres de los controles normales y a la poca precisión que tiene dicha puntuación en el diagnóstico de la asfixia perinatal ${ }^{3}$. Diversos estudios han demostrado que durante el trabajo de parto se produce aumento de la concentración de ácido láctico, materno $y$ fetal $^{7-9}$, que puede alterar el equilibrio ácido básico en la primera hora de vida. Hasta la fecha no se han publicado estudios que comparen los valores de $\mathrm{pH}$ en la arteria umbilical con y sin trabajo de parto y que

1. Departamento de Pediatría, Unidad de Neonatología.

2. Departamento de Obstetricia y Ginecologia. Pontificia Universidad Católica de Chile. analicen los factores determinantes del $\mathrm{pH}$ en el momento de nacer.

El objetivo de este trabajo es describir los valores de $\mathrm{pH}, \mathrm{PO}_{2}, \mathrm{PCO}_{2}$ y exceso de base, medidos simultáneamente en arteria y vena umbilical en una población obstétrica rigurosamente controlada, mediante monitoría electrónica durante el parto, estudiar el efecto del trabajo de parto sobre el equilibrio ácido básico fetal y determinar valores normales que posteriormente sirvan de patrones de comparación con poblaciones patológicas.

\section{MATERLAL Y METODOS}

Se estudiaron 19 recién nacidos (RN) de término nacidos por parto vaginal y 19 RN término nacidos por cesárea electiva, sin trabajo de parto previo. Se incluyeton solamente embarazos únicos, sin morbilidad materna a fetal, con eestaciones superiores a 37 semanas y menores de 42 semanas, según fecha confiable de última regla o ecografía precoz o ambas; con indemnidad de la unidad letoplacentaria en el momento de la resolución del parto (monitoría electrónica normal y líquido amniótico claro en amnioscopía). Se excluyeron los RN en que hubo alteración de los latidos cardiofetales, circulares de cordón y fórceps. En caso de anestesia sólo se aceptó la epidural. Las madres no recíbieron analgésicos ni sedantes. En los nacidos por vía vaginal se exigjó que el trabajo de parto fuera espontáneo, el periodo de dilatación menor de 10 horas y el expulsivo igual o menor de 20 minutos. En los nacidos por cesárea, que èsta fue- 
se electiva por desproporción pelvifetal, sin trabajo de parto previo. Se incluyeron RN cuyos pesos de nacimiento estaban sobre 2500 y menos de $4250 \mathrm{~g}$, Apgar $>8$ en el primer minuto $y>9$ a los 5 minutos de nacer $y$ cuyo examen físico fuese normal.

En el momento de nacer se tomó una muestra de sangre de arteria y otra de vena umbilical en un segmento de cordón pinzado en ambos extremos. Las muestras se obtuvieron con jeringas de plástico heparinizadas conservadas en hielo y fueron analizadas dentro de 10 a 15 minutos de extraídas, mediante un artalizador de gases automático ( $\mathrm{ABL}$, Radiometer, Copenhagen), que mide $\mathrm{pH}_{4} \mathrm{pCO}_{2}$ y $\mathrm{pO}_{2}$ directamente, calculando el exceso de base según pH y $\mathrm{PCO}_{2}$ de la muestra.

Posteriormente los RN fueron observados en un ambiente térmico neutral durante 6 horas y examinados por un neonatólogo sin conocímiento previo de los antecedentes, ni del resultado de los gases de cordón, constatándose ausencia de anormalidad clínica. Todos fueron dados de alta a los 3 a 4 días de edad, en buemas condiciones

El estudio se realizó en el Servicio de Obstetricia, Ginecología y Perinatología conjuntamente con la Unidad de Neonatología del Hospital Clínico de la Pontificia Universidad Católica de Chile.

Los datos fueron ingresados a un computador y analizados por el Servicio de Consultoría Estadística de dicha universidad. Se utilizó la prueba de Student para la diferencia de medias $y$ análisis de correlación $y$ regresión lineal para determinar asociación entre variables. Valores de $\mathrm{p}<0,05$ se consideraron estad ísticamente significativos.

\section{RESULTADOS}

Se estudiaron $38 \mathrm{RN}$ que reunian las caracteristicas anteriormente indicadas. 19 nacieron por partos vaginales y 19 por cesáreas electivas debidas a desproporción cefalopélvica, sin trabajo de parto previo. El peso de los partos vaginales fue de $3.492 \mathrm{~g} \pm 484 \mathrm{~g}$ y su edad gestacional $39,0 \pm 1,5$ semanas; el peso de los nacidos por cesárea fue $3.312 \mathrm{~g} \pm 530 \mathrm{~g}$ y la edad gestacional de 38,70 $\pm 1,04$ semanas $(\mathrm{P} n)(\overline{\mathrm{X}} \pm \mathrm{DE})$.

En ambos grupos la distribución de los valores de pH fue de tipo normal. Para los partos vaginales $95 \%$ de las observaciones se encuentran entre pH 7,11 y 7,35, mediana 7,24. En los nacidos por cesáreas los valores de $\mathrm{pH}$ se ubican entre 7,21 y 7,33 , mediana 7,29 .

Los valores de la media $\pm 1 \mathrm{DE}$ para $\mathrm{pH}, \mathrm{pO}_{2}$, $\mathrm{pCO}_{2}$ y exceso de base medidos en la arteria $y$ vena umbilical se muestran en la tabla 1 .

En general se observó marcada variabilidad en los valores de $\mathrm{pCO}_{2}$ y $\mathrm{pH}$ en los niños nacidos por vía vaginal, mientras que en los nacidos por vía cesárea éstos fueron más homogéneos. El $\mathrm{pH}$ sanguíneo de arteria umbilical fue significativamente más bajo $(7,23 \pm 0,06)$ en los $R N$ cuya

\section{Tabla 1}

Valores de $\mathrm{pH}, \mathrm{pCO}_{2}, \mathrm{pO}_{2}$ y exceso de base en sangre de arteria y vena umbilical de recién nacidos, según la via de parto.

\begin{tabular}{|c|c|c|c|c|c|c|c|c|}
\hline \multirow[b]{3}{*}{$\mathrm{pH}$} & \multicolumn{3}{|c|}{$\begin{array}{c}\text { Partos vaginales } \\
\text { N: } 19\end{array}$} & \multicolumn{5}{|c|}{$\begin{array}{l}\text { Cesáreas electivas } \\
\mathrm{N}: 19\end{array}$} \\
\hline & \multicolumn{2}{|c|}{ (media $\pm 1 \mathrm{DE}$ ) } & (tango) & \multicolumn{2}{|c|}{ (media $\pm 1 \mathrm{DE})$} & \multicolumn{3}{|c|}{ (rango) } \\
\hline & A & $7,23 \pm 0,06$ & $(7,11-7,34)^{*}$ & $7,27 \pm$ & 0,03 & $(7,20$ & $0-7,3$ & 1)* \\
\hline & $\mathrm{v}$ & $7,29 \pm 0,04$ & $(7.23-7.40)$ & $7,32 \pm$ & 0,03 & $(7,23$ & $3-7,3$ & \\
\hline $\mathrm{pCO}_{2}$ & A & $45,41 \pm 11,66$ & $(23,6-64,9)$ & $46,74 \pm$ & 4,71 & $(39,5$ & -56 & ) \\
\hline $\mathrm{mmHg}$ & $\mathrm{V}$ & $38,26 \pm 7,25$ & $(21,9-52,7)$ & $38.99 \pm$ & 5,38 & $(32,7$ & -54 & ) \\
\hline $\mathrm{pO}_{2}$ & A & $18,83 \pm 6,71$ & $(7,3-32,5)$ & $19,05 \pm$ & 6,78 & $(5,6$ & -34 & ) \\
\hline $\mathrm{mmHg}$ & $v$ & $27,15 \pm 5,22$ & $(18,2-34,1)$ & $28,46 \pm$ & 6,80 & $(16,8$ & -40 & 3 \\
\hline EB & $A$ & $-8,49 \pm 2,86$ & $(-3,6-12,7)$ & $-5,24 \pm$ & 2,39 & $(-2,7$ & $-10,5$ & )$^{* *}$ \\
\hline $\mathrm{mEg} / 1$ & $\mathrm{v}$ & $-6,50 \pm 4,00$ & $(-2,2-12,5)$ & $-4,92 \pm$ & 1,60 & $(-3,3$ & $-9,3$ & 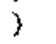 \\
\hline
\end{tabular}

$* P<0,01$

** $P<0,001$

$A=$ arteris

$\mathrm{V}=$ vena 
madre tuvo trabajo de parto previo que en los nacidos por cesárea electiva $(7,27 \pm 0,03) \mathrm{p}<0,01$. No se observaron diferencias en el $\mathrm{pH}$ de sangre de vena umbilical de ambos grupos.

El exceso de base fue significativamente mayor en la arteria umbilical de los nacidos por parto vaginal $(8,49 \pm 2,86 \mathrm{mEq} / 1)$ que en los nacidos por cesárea $(5,24 \pm 2,39 \mathrm{mEq} / 1) \mathrm{p}<$ 0,001 .

No hubo diferencias en los promedios de $\mathrm{PO}_{2}$ y $\mathrm{PCO}_{2}$ de sangre de las arterias y de las venas umbilicales entre ambos grupos. En los partos vaginales la variable que mejor se correlacionó con el $\mathrm{pH}$ arterial fue la $\mathrm{PCO}_{2}(\mathrm{r}: 0,72)$ segujdos por la $\mathrm{PO}_{2}$ (r: 0,67 ) p $<0,01$. En los niños nacidos por cesáreas electivas el pH se correlacionó mejor con el exceso de base ( $r: 0,73$ ) seguido por el nivel de $\mathrm{PO}_{2}(\mathrm{r}: 0,56) \mathrm{p}<0,01$.

\section{DISCUSION}

El trabajo de parto ocurre por cambios fisiológicos que modifican el equilibrio ácido básico materno y fetal ${ }^{7,8,10}$. Se produce leve aumento de la concentración de ácido láctico materno, secundario al aumento del trabajo muscular, a los periodos de hipoventilación e hipocapnia $y$ al efecto de la secreción de catecolaminas maternas $^{7,8} ; \sin$ embargo, este fenómeno no se traduce en mayor acidosis fetal, dado que el ácido láctico no cruza la barrera placentaria ${ }^{10}$. En el feto también se ha observado aumento de la concentración de ácido láctico, asociado a aumento de la relación lactato/piruvato, lo que indica que este fenómeno es producto de glicólisis anaeróbica secundaria a hipoxia tisular.

La mayor acidosis que presentaron los niños nacidos por la vía vaginal corrobora esta hipótesis. La condición clínica y la evolución posterior de ellos fue normal, comparable con los nacidos por la vía cesárea, en que no hubo trabajo de parto previo; por lo tanto esta acidosis debe ser considerada fisiológica y tomada en cuenta en la interpretación del $\mathrm{pH}$ de arteria umbilical.

Los valores normales de $\mathrm{pH}$ en la arteria umbilical, publicados en la literatura, varian entre 7,23 y $7,26^{1,4,5,8,12}$. En la mayoría de esos trabajos se han incluido pacientes nacidos con y sin trabajo de parto $y$ se ha tomado como referencia la media \pm 1 desviación estándar, considerándose valores anormales del $\mathrm{pH}$ los inferiores a $7,20^{1}$. Creemos que debido a la gran variabilidad del $\mathrm{pH}$ de la sangre arterial umbilical es preciso usar la media $\pm 2 \mathrm{DE}$, pues cubre $95 \%$ de las observaciones, evitando de esta manera valores falsamente positivos.

Basados en nuestros resultados, consideramos que el rango de $\mathrm{pH}$ normal para la arteria umbilical debiera ser estimado en $7,23 \pm 2 \mathrm{DE}(7,11$ a 7,35 ) en los partos vaginales y 7,27 $\pm 2 \mathrm{DE}$ $(7,21$ a 7,33$)$ para los nacidos por cesáreas electivas.

La toma de muestra de un segmento de cordón umbilical es un método no invasivo y fácil, que permite evaluar el estado ácido básico del feto y correlacionarlo con la condición clinica del recièn nacido, especialmente cuando hay antecedentes de sufrimiento fetal agudo o Apgar bajo. Sin embargo, para interpretar correctamente los valores de $\mathrm{pH}$, es importante tomar en cuenta los antecedentes perinatales y la vía de parto.

\section{RESUMEN}

Se estudió la influencia del trabajo de parto en el equilibrio ácido básico del RN, comparando los valores de $\mathrm{pH}, \mathrm{pCO}_{2}, \mathrm{paO}_{2}$ y exceso de base, de arteria umbilical, en 19 recién nacidos por vía vaginal y 19 nacidos por cesárea electiva, sin trabajo de parto previo. El pH fue significativamente más bajo en los nacidos por partos vaginales $(7,23 \pm 0,06)$ que por cesáreas electivas $(7,27 \pm 0,03) \mathrm{p}<0,01$. El $\mathrm{pH}$ tuvo mejor correlación con las concentraciones de $\mathrm{CO}_{2}$ en los partos vaginales $(r: 0,72) p<0,01$ y con el de exceso de base en los nacidos por cesárea (r: 0,73$) \mathrm{p}<0,01$. El valor de $\mathrm{pH}$ de la arteria umbilical del $\mathrm{RN}$ es influenciado por el trabajo de parto. Estimamos que deben considerarse anormales los $\mathrm{pH}$ arteriales de cordón inferiores a 7,11 (media - 2 DE) en parto vaginal y los menores que 7,21 en cesáreas electivas sin trabajo de parto.

(Palabras claves: pH arteria umbilical, recién nacidos, parto vaginal, cesárea electiva.)

\section{REFERENCIAS}

1. D'Souze S.w., Black P., Cadman J.: Umbilical venous blood $\mathrm{pH}$ : a useful aid in the diagnosis of asphyxia at birth. Arch Dis Child, 1983; 38: 15-19. 
2. Fields L.M., Entman S.S., Boehm F.H.: Correlation of the one-minute Apgar Score and pH value in umbilical arterial blood. South Med J 1983; 76: 1477-1479.

3. Sykes G.S., Johnson P., Ashworth F. et al.: Do Apgar score indicate asphyxia?, Lancet 1982; j: 494-496.

4. Chow $S \cdot N$. Chen H.Y.: Maternal and Fetal Blood Acid-Base status at delivery. I Formosan Med Assoc 1982; 81: 674-685.

5. James L.S., Weistrot I.M., Prince C.E.: The AcidBase Status of human infants in relation to birth asphyxia and the onset of respiralion. J Pediatr $1958 ; 52: 379-393$.

6. Clark B.R. Cooper J.O. Stephens S.R.: Neonatal Acid-Base Studies. Effect of normal labor and obstetric manipulation Obstet Gynecol 1969; 33: 23-29.

7. Low J.A., Pancham S.R, Worthatgton D.: AcidBasc, Lactante, and Pyruvate Characteristics of the normal obstetric patient and fetus during the intrapartum period. Am J Obstet Gynecol 1974; 120: $862-867$.
8. Low J.A.. Pancham S.R., Piercy W.N.: Maternal and Fetal Lactate characteristic during Labot and Delivery. Lactate in Acute Conditions in International symposium on lactate in acute conditions. Ed $H$ Bossart and C Perret, Lausanne. S Karger 1978: 29-47.

9. Bratteby I.E., Swanstrom $S$ : Arterial pyruvate concentration in the normal and asphyxiated newborn infant, in Lactate in acute conditions, in International Symposium on Lactate in Acute conditions. Ed $\mathbf{H}$ Bossert and C Perret, Lausartne. S Karger 1978; 56-68.

10. Roth G.: The Placental transfer of gases and acids, Arch Dis Child 1962;37:366-370.

11. Hacobson, L., Gardmork and Rooth G.: Fetal bradycardia after Paracervical Block caused by rapid maternal $\mathrm{pCO}_{2}$ and $\mathrm{pH}$ changes, Excerpta Medica, International Congress Series 1973; 279: 83-87.

12. Wible, J.L., Petrie, R.H., Koons A.: The clinical use of umbilical cord acjd-base determinations in Perinatal Survejllance and Maragement. Clin Perinatol 1982; 9: 387-397. 